\title{
Young children reorient by computing layout geometry, not by matching images of the environment
}

\author{
Sang Ah Lee • Elizabeth S. Spelke
}

Published online: 19 November 2010

(C) Psychonomic Society, Inc. 2010

\begin{abstract}
Disoriented animals from ants to humans reorient in accord with the shape of the surrounding surface layout: a behavioral pattern long taken as evidence for sensitivity to layout geometry. Recent computational models suggest, however, that the reorientation process may not depend on geometrical analyses but instead on the matching of brightness contours in 2D images of the environment. Here we test this suggestion by investigating young children's reorientation in enclosed environments. Children reoriented by extremely subtle geometric properties of the 3D layout: bumps and ridges that protruded only slightly off the floor, producing edges with low contrast. Moreover, children failed to reorient by prominent brightness contours in continuous layouts with no distinctive 3D structure. The findings provide evidence that geometric layout representations support children's reorientation.
\end{abstract}

Keywords Reorientation · Navigation · Image-matching · Layout geometry

What are the origins of human geometrical intuitions (e.g., Plato, 380/1949)? For centuries, psychologists and neuroscientists have proposed that geometrical concepts arise from the representations that guide navigation (e.g., von Helmholtz, 1885/1962). The cognitive map hypothesis posited that navigating animals form a coherent Euclidean representation of the environment and the objects within it by computing the geometric structure of their own movements (O'Keefe \& Nadel, 1978; Tolman, 1948). This idea has been undermined, however, by evidence that suggests that rather than encoding

\section{S. A. Lee $(\bowtie) \cdot$ E. S. Spelke}

Department of Psychology, Harvard University,

33 Kirkland Street,

Cambridge, MA 02138, USA

e-mail: sangah@gmail.com
Euclidean relationships, animals and humans learn routes between locations and recognize goal locations by matching stored, viewpoint specific, two-dimensional "snapshots," of those locations (Cartwright \& Collett, 1982; Foo, Duchon, Warren, \& Tarr, 2007; Mackintosh, 2002; Wehner \& Menzel, 1990; for review, see Shettleworth, 2010).

In the past few decades, however, the thesis that truly geometrical representations underlie navigation processes has been reinvigorated by studies of animals' spontaneous encoding of the geometry of the surrounding, extended surface layout. In one set of studies, rats discovered the location of food in a geometrically distinctive environment, then were disoriented by slow turning, and finally were allowed to reorient and search for the food. These animals, and other species including ants, fish, birds, and humans, reestablished their sense of orientation by relying wholly or in part on the metric shape of the surrounding surface layout (Cheng, 1986; Cheng \& Gallistel, 1984; Wystrach \& Beugnon, 2009; for review, see Cheng \& Newcombe, 2005). When the layout was rectangular, they divided their search between the correct corner and the geometrically equivalent opposite corner, consistent with the lengths and sense relationships between the room's borders. Other studies, using neurophysiological and neuroimaging methods, provided evidence that oriented rats and humans automatically encoded both their own position and the positions of objects relative to the borders of the navigable layout, largely irrespective of the presence of freestanding objects or distinctive surface colors or textures (Doeller \& Burgess, 2008; Lever, Wills, Cacucci, Burgess, \& O'Keefe, 2002; O'Keefe \& Burgess, 1996). Rodents and humans also can learn the metric relations between separated objects, but this learning appears to depend on a different navigation process, modulated by training and attention (Benhamou \& Poucet, 1998; Doeller \& Burgess, 2008; Gibson, Wilks, \& Kelly, 2007; Pecchia \& Vallortigara, 2010; Twyman, Friedman, \& Spetch 2007; see Cheng \& Newcombe, 
2005). Although multiple independent processes contribute to spatial behavior, these findings suggest that navigation depends in part on a spontaneous analysis of surface layout geometry (Gallistel, 1990; Wang \& Spelke, 2002).

More recently, however, the suggestion that truly geometrical representations are involved in navigation has been challenged once again by the observation that enclosed rectangular environments produce salient brightness contours in images of the array (Cheng, 2008). In computational studies, processes for matching brightness contour maps of the perceived and remembered environment were shown to account for the primary findings of experiments on reorientation (Sheynikhovich, Chavarriaga, Strösslin, Arleo, \& Gerstner, 2009; Stürzl, Cheung, Cheng, $\&$ Zeil, 2008). It has been suggested that image-matching processes at two distinct spatial scales also account for disoriented insects' search performance (Wystrach \& Beugnon, 2009). If reorientation is based solely on retinal image-matching processes, however, then these navigation processes would fail to capture the geometric properties and relations at the center of human geometrical intuitions.

Curiously, however, image-matching and geometrical congruence-finding processes have not been contrasted empirically, in any species, because sensitivity to layout structure has only been tested in environments with a large and highly distinctive shape, producing a marked array of brightness borders. In four experiments, therefore, we tested young children's reorientation in rectangular arrays either with geometrically distinctive 3D surface layouts producing small and subtle brightness borders (experiments 1 and 2), or with geometrically uninformative surface layouts containing geometrically informative, highly pronounced brightness borders (experiments 3 and 4). If imagematching processes underlie children's reorientation, then they should perform best in experiments 3 and 4, which present the strongest contrast borders. If children reorient by the shape of the extended surface layout, then they should perform best in experiments 1 and 2, in which the surface layout has a subtle but distinctive shape.

\section{General methods and preliminary analyses}

Participants Children $(n=64)$ aged 38-51 months (mean 44 months) took part in the experiments: eight boys, eight girls each in experiments $1 \& 2$; seven boys, nine girls each in experiments $3 \& 4$. All resided in the Boston area.

Testing room Experiments took place in a circular room (3.7-m diameter) made of white curved panels and a light grey floor. Centered in the ceiling were a hidden camera and microphone with concentrically surrounding lights. All experiments presented four hiding places arranged in a
$1.2 \times 1.8 \mathrm{~m}$ rectangular array at the room's center, following past studies (e.g., Hermer \& Spelke, 1996; Learmonth, Nadel, \& Newcombe, 2002). Other room features varied across experiments and are described below.

Procedure Children were tested by an experimenter inside the room, while the parent and an observer watched the study via closed-circuit video outside the room. As the child watched from the array's center, the experimenter pointed out the four hiding locations and then placed a sticker underneath one of them. Then the child was blindfolded and turned slowly until he or she was disoriented, as evidenced by a failure to point correctly to the door of the chamber (typically 3-4 rotations). Finally, the experimenter faced the child towards one of four predetermined directions, stood behind the child, removed the blindfold, and encouraged the child to find the sticker. The experimenter stood still, looking directly at the child, without shifting her gaze to any of the hiding locations, until the child lifted the cover (e.g., flap/container) at one of the hiding places. After the child's first search, the experimenter retrieved the sticker, if necessary, gave it to the child, and introduced a new sticker for the next trial.

Design A session consisted of four trials at a constant hiding location; equal numbers of children were tested at each location. Following disorientation, the child faced midway between a different pair of adjacent hiding places on each trial; facing direction order was counterbalanced across subjects.

Analyses and preliminary findings First searches were recorded from video by naive observers. To determine whether the disorientation procedure was effective, and whether the displays were devoid of inadvertent cues, searching at the correct location was compared to searching at the geometrically equivalent opposite location. These search rates did not differ in any of the experiments (all $t \mathrm{~s}(15)<1$, n.s.). To assess children's sensitivity to the rectangular geometric structure, the proportion of geometrically correct searches (i.e., at either the correct or the diagonally opposite location) was tested against chance (50\%), using a two-tailed $t$ test; these analyses are reported below. To test for sex differences in performance, the rates of geometrically correct searches were compared for boys and girls. There were no sex differences in any study (all $t \mathrm{~s}(15)<$ 1, n.s.) and no trends favoring either sex (across the four experiments, $59 \%$ geometric search for girls, $62 \%$ geometric search for boys, $t(62)<1$, n.s.).

\section{Experiment 1}

We first presented children with a rectangular frame composed of white rods just $2 \mathrm{~cm}$ in height and width, 
a) Experiment 1 Borders

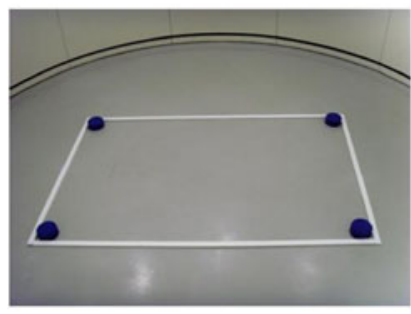

e)

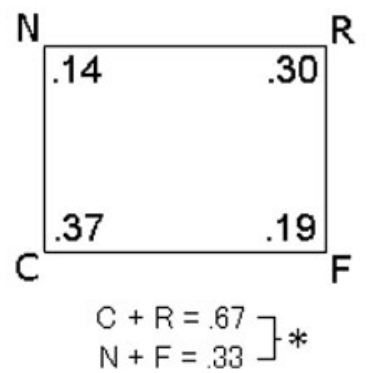

b) Experiment 2 Curved Hills

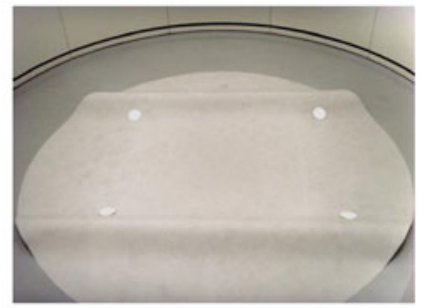

$\mathrm{N}$

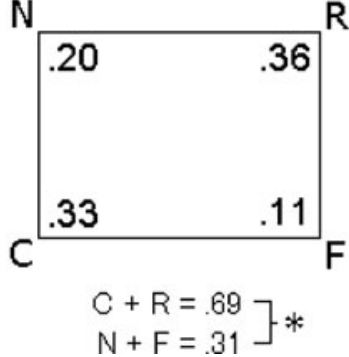

c) Experiment 3 20 Form
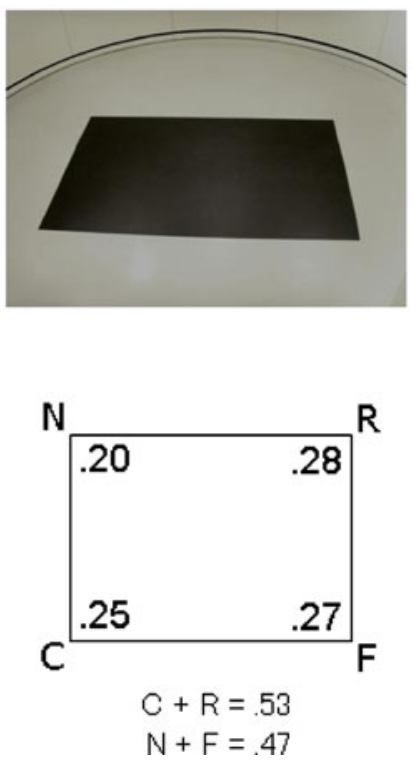

d) Experiment 4 Columns
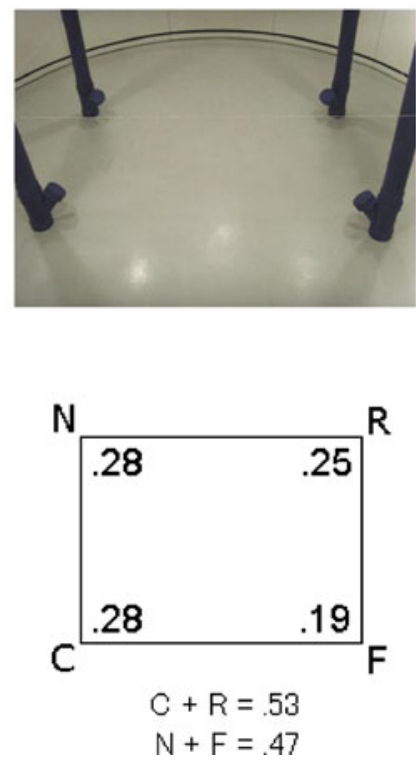

Fig. 1 Photographs of the rectangular arrays within the cylindrical testing space used in a experiment 1 , b experiment 2, c experiment 3 , and $\mathbf{d}$ experiment 4 . In $\mathbf{a}$ and $\mathbf{b}$, the contrast of the photographs was enhanced to increase the visibility of the structures. e Proportions of children's first search at the correct corner $(\mathbf{c})$, the corner rotationally

fixed to the light floor (Fig. 1a). Four blue cylindrical cups (10 $\mathrm{cm}$ wide, $2 \mathrm{~cm}$ high), inverted and placed in the corners of the frame, served as hiding places. Because the luminance change from the topmost surface of the white frame $\left(80.6 \mathrm{~cd} / \mathrm{m}^{2}\right)$ to the darkest part of the shadow directly adjacent to it $\left(57.2 \mathrm{~cd} / \mathrm{m}^{2}\right)$ was small $\left(\right.$ Contrast $^{1}=$ 0.17 ), the frame's borders created only subtle contrast edges in the array.

Results Children searched at the correct and opposite corners more often than the other two corners $(67 \%$ geometric search, Cohen's $d=0.61$, two-tailed $t(15)=$ $2.71, p=.016)$. Performance in the first two trials $(69 \%)$ did not differ significantly from performance in the last two trials $(64 \%), t<1$, n.s., indicating no improvement over trials. Children therefore reoriented successfully in this experiment, relying on subtle perturbations in the $3 \mathrm{D}$ surface layout. Nevertheless, image-matching theories might explain the present findings, because the display included four hiding containers at its corners and the rectangular edges of the frame cast shadows at the borders of the array. The next experiment tested further for

\footnotetext{
${ }^{1}$ We compute contrast as $(H-L) /(H+L)$, where $H$ and $L$ denote the luminance of the higher and lower regions, measured from the child's eye height at the room's center.
}

symmetric to the correct location $(R)$, the nearby incorrect corner $(N)$, and the farther incorrect corner $(F) . \mathrm{C}+\mathrm{R}$ indicates the total geometrically correct searches, and $\mathrm{N}+\mathrm{F}$ indicates the total geometrically incorrect searches. Because hiding places varied across subjects, all data have been rotated into alignment

children's ability to reorient by subtle layout geometry in arrays with no hiding containers or rectilinear edges creating discontinuities in surface brightness.

\section{Experiment 2}

Experiment 2 altered the homogeneous cylindrical environment by centering two parallel smooth bumps on the floor. These bumps, produced by cylindrical pipes $(10 \mathrm{~cm}$ wide, $1.9 \mathrm{~m}$ long, $1.25 \mathrm{~m}$ apart) beneath a circular piece of white vinyl flooring ( $2.4 \mathrm{~m}$ diameter), perturbed the shape of the ground surface but produced no discontinuous changes in its illumination. Four 2D circular disks $(10 \mathrm{~cm}$ wide) of the same flooring material formed a rectangular array at the inner sides of the bumps and served as hiding locations (Fig. 1b). The luminance change from the lightest point on the curved bump $\left(81.0 \mathrm{~cd} / \mathrm{m}^{2}\right)$ to the darkest part of the curved surface directly adjacent to the bump $\left(72.3 \mathrm{~cd} / \mathrm{m}^{2}\right)$ was small $($ contrast $=0.06)$.

Results Children restricted their search to the two geometrically correct locations $(69 \%$ geometric search, Cohen's $d=0.88$, two-tailed $t(15)=3.50, p=.003)$. Performance in the first two trials $(72 \%)$ did not significantly differ from 
performance in the last two trials $(66 \%), t<1$, n.s. Children therefore reoriented by a subtle, curved perturbation to the 3D shape of the room's surface layout, despite the absence of sharp brightness discontinuities in this array.

The findings of experiments 1 and 2 are not easily explained by image-matching theories, but they fail to rule out such theories conclusively. Because 3D surface layouts do produce small brightness borders, children's success could be explained either by a highly sensitive imagematching process or by a process for analyzing surface layout geometry. The crucial evidence to distinguish these theories would come from a comparison of children's sensitivity to the shapes formed by extended surfaces, on one hand, and the shapes formed by discontinuities in surface lightness on the other. Image-matching theories predict that children will reorient by any array presenting brightness changes that are as large as, or larger than, the brightness changes in experiments 1 and 2 .

Previous experiments provide suggestive evidence against this prediction. In particular, children successfully reorient by a rectangular arena with $30-\mathrm{cm}$-high walls but not by a $2 \mathrm{D}$ rectangular line figure of the same size (Lee \& Spelke, 2008). Moreover, children reorient by a circular arena whose shape is perturbed by two dark columns that stand flush with its walls, but not by dark 2D patches of the same size (Lee \& Spelke, 2010). Nevertheless, neither of these studies measured the brightness borders in the array, and neither compared children's responses to prominent brightness borders with their response to subtle perturbations in 3D surfaces. Experiments 3 and 4 were undertaken to allow this comparison.

\section{Experiment 3}

Because past research had already tested children's reorientation by an outline rectangle whose brightness contrast would be diminished at low resolution (Lee \& Spelke, 2008), experiment 3 presented children with a solid 2D black rectangle centered on the light floor (Fig. 1c). The rectangle was made of black vinyl contact paper, with black vinyl flaps (10 cm wide) at its corners serving as hiding places. The luminance measures were $60.4 \mathrm{~cd} / \mathrm{m}^{2}$ (floor) and $8.0 \mathrm{~cd} / \mathrm{m}^{2}$ (black rectangle) $($ contrast $=.77)$.

Results Children restricted their search to the four corners of the rectangle ( $100 \%$ of trials), showing that they detected this form and remembered its relevance for the search task. Nevertheless, children chose among these corners at random $(53 \%$ geometric search, Cohen's $d=0.21, t(15)=$ $0.42, p=.684)$. Despite the high contrast between the rectangular form and the surrounding environment, children failed to reorient by the shape of this form.
Children's failure with salient 2D features and success with subtle 3D perturbations in the ground surface do not accord with the predictions of image-matching theories. Nevertheless, a modification of those theories could account for the present findings. Because the arrays in experiments 1 and 2 projected upward from the floor, they introduced some constraint on children's locomotion, whereas the array in experiment 3 did not. Although children attended to the $2 \mathrm{D}$ patch enough to confine their search to its corners, they may have attended more to the geometric shapes of the $3 \mathrm{D}$ projections to avoid tripping over them. Image-matching processes therefore may underlie children's navigation in all these studies, but children may engage these processes more reliably when their locomotion is constrained. Experiment 4 tested this possibility.

Experiment 4 was undertaken for a further reason. The most salient 2D image information presented in past studies of animal navigation has come not from surface contrast on the floor but from large freestanding columns, projecting upward from the ground and serving as vertical markers of the space (e.g. Cartwright \& Collett, 1982). In past research, however, freestanding objects have not served as reliable guides to orientation in children (Gouteux \& Spelke, 2001; Lee, Shusterman, \& Spelke, 2006; Lee \& Spelke, 2008) or rats (Cressant, Muller, \& Poucet, 1997; Zugaro, Berthoz, \& Wiener, 2001) although animals can learn to use them with training (Benhamou \& Poucet, 1998; Gibson et al., 2007; Pecchia \& Vallortigara, 2010). Accordingly, the final experiment tested children's disoriented search within a rectangle composed of four tall, dark columns against the white surroundings.

\section{Experiment 4}

Experiment 4 presented children with four dark blue columns $(180 \mathrm{~cm}$ tall, $10 \mathrm{~cm}$ wide, with hiding well at $15 \mathrm{~cm}$ ) in a rectangular array (as in Lee \& Spelke, 2008). To restrict children's movement without introducing any continuous surface into the array, columns were connected together by a beige cord ( $2 \mathrm{~mm}$ thick) at eye level $(80 \mathrm{~cm})$ for half the children and near the ankle $(15 \mathrm{~cm})$ for the others (Fig. 1d). Luminance measures were $58.7 \mathrm{~cd} / \mathrm{m}^{2}$ (floor) and $6.6 \mathrm{~cd} / \mathrm{m}^{2}$ (column) (contrast $\left.=.80\right)$.

Results All children stayed within the space delimited by the cord. As in experiment 3, children confined their search to the columns $(100 \%$ of searches), indicating that they detected them and recognized their relevance to the search task. Nevertheless, children chose among the columns at random (53\% geometric search, Cohen's $d=0.24, t(15)=0.46$, $p=.652$ ). There was no difference in performance between children presented with the cord near eye level vs. near the 
ankle $(t(14)<1)$. Children therefore failed to reorient by the array of columns, despite the cord that constrained their motion and despite contrast between the columns and the surrounding environment that was more than 13 times greater than that of experiment 2 .

In a final analysis, we compared performance in the experiments with 3D surface layouts of low contrast (experiments 1 and 2) to performance in the experiments with 2D borders or 3D freestanding objects of high contrast (experiments 3 and 4). Reorientation by the continuous 3D surface layouts showed a significant advantage over reorientation by patterns of salient brightness contrast, $F(1,60)=5.21, p=.025$ : an effect opposite in direction to that predicted by image-matching theories (Sheynikhovich et al., 2009; Stürzl et al., 2008), and consistent with the thesis that children reorient by subtle surface layout geometry.

\section{General discussion}

The present findings provide evidence that children's navigation depends on geometrical computations applied to representations of the $3 \mathrm{D}$, continuous surface layout. Children are highly sensitive to the geometrical structure of small perturbations in the surface layout involving either abrupt or gradual changes in surface orientation. Strikingly, children's successful reorientation by the shape of the layout contrasts with their failure to reorient by the shape of the $2 \mathrm{D}$ rectangle or array of freestanding columns, despite the strong contrast borders in those arrays. Importantly, children in experiments 3 and 4 reliably encoded and remembered the features of the hiding places and used them to constrain their search to a $2 \mathrm{D}$ corner or column. Nevertheless, children did not reorient by the relative distances and directions of these environmental features.

These results cast doubt on image-matching theories of reorientation (Cheng, 2008; Sheynikhovich et al., 2009; Stürzl et al., 2008; Wystrach \& Beugnon, 2009), as neither the prominence of brightness edges in the array nor the size and salience of objects predicted children's patterns of success and failure. In contrast, the results accord with the original theory of Cheng (1986) and Gallistel (1990), as well as the more recent theories of, and evidence for, the primacy of extended surface layouts in animal navigation (Doeller \& Burgess, 2008; Lever et al., 2002; O'Keefe \& Burgess, 1996; Solstad, Boccara, Kropff, Moser, \& Moser, 2008). Indeed, the present findings go beyond past evidence in revealing that navigating children are highly sensitive to small asymmetries in surface layout geometry. This high sensitivity suggests that navigating humans will be able to maintain and recover their sense of orientation in a very wide range of environments, both open and enclosed, for natural environments will seldom contain symmetries at the subtle scales tested in these experiments (Gallistel, 1990).

In casting doubt on image-matching theories, the present findings converge with those of three recent studies of reorientation in children (Huttenlocher \& Lourenco, 2007; Lee \& Spelke, 2008, 2010). Huttenlocher and Lourenco (2007) presented 2.5-year-old children with a square arena whose alternating walls differed in color (blue vs. red) or patterning (circles vs. crosses in one experiment and circles vs. gray walls in another experiment). Children failed to reorient by any of these image features, contrary to imagematching theories. Indeed, the sole image manipulation that supported children's reorientation in Huttenlocher and Lourenco's experiments was one in which the alternating walls presented circles that were large and sparse or small and dense. This condition presents less salient image changes than those of their other studies, but it may influence children's perception of the room's shape through the depth cue of relative size (Lee, Winkler-Rhoades, \& Spelke, 2010).

The present findings provide further evidence for a dissociation between the process by which navigators reestablish their own orientation with respect to the external environment and the process by which they learn and relocate hidden objects relative to stable visible landmarks. Oriented animals are highly predisposed to use surface markings like those in experiment 3 , and large objects like the columns in experiment 4 , to mark significant locations (respectively, Pearce, Graham, Good, Jones, \& McGregor, 2006; Cartwright \& Collett, 1982). The children in experiments 3 and 4 appeared to do this as well, using the 2D corners and columns as direct guides to the hidden object. Because all four corners or columns were similar, however, local images did not distinguish one corner or column from another. To do so, children needed to encode the geometrical structure of the rectangular arrays, and they did so successfully only when that structure was part of the continuous, 3D surface layout.

The present findings do not reveal whether geometric computations also underlie reorientation by other animals. While there is increasing evidence for homologies between the navigation systems in rats and humans (e.g., Doeller, Barry, \& Burgess, 2010), one set of findings from neurophysiological studies of rodents is often cited as strong evidence for image-matching computations of orientation (e.g., Sheynikhovich et al., 2009), and therefore conflicts with the present findings. When rats freely explore a square or cylindrical environment containing a single cue card that contrasts in brightness with its surroundings (such as a white card against a black background), neurons in the hippocampus and surrounding cortex whose firing is 
modulated by the animal's spatial position and heading change their firing predictably with the movement of the cue card to a new location along the walls of the arena (Knierim, Kudrimoti, \& McNaughton, 1995; Taube, Muller, \& Ranck, 1990). These place and head-direction fields persist in darkness, indicating that they reflect the animal's represented position and heading, not its current visual experience (Goodridge, Dudchenko, Worboys, Golob, \& Taube, 1998; Quirk, Muller, \& Kubie, 1990). Moreover, changes in the environment that alter the animal's representation of its position with respect to the environment cause ensembles of place cells and nearby border cells to change their firing in unison (Lever et al., 2002; Solstad et al., 2008).

All these findings suggest that the animal uses the cue card to specify and reestablish its own position, but why does a cue card have this effect? Because a white card upon a black background produces a large brightness edge, but only a subtle geometric protrusion, investigators have assumed that the alignment of place and head-direction cells to the position of such a card is caused by its moving contrast borders, not by the asymmetry in the shape of the arena. Nevertheless, because a cue card often also has a discernible thickness, the present findings raise a different possibility: place and head-direction cells may respond only to the distances and directions of environmental borders, and their sensitivity to subtle changes in the $3 \mathrm{D}$ layout may be high enough to capture the thickness of the cue card. Both behavioral and neurophysiological experiments with other animals, using completely 2D landmarks and subtle 3D surface layouts, are needed to test this hypothesis.

However this controversy over animal navigation is resolved, the present experiments show that processes guiding navigation in children have truly geometric content. These findings therefore provide a new basis for testing an old idea: that human knowledge of abstract geometry may be rooted in processes guiding navigation (O'Keefe \& Nadel, 1978; Tolman, 1948; von Helmholtz, 1885/1962). More specifically, the geometrical computations underlying young children's navigation may be a source of uniquely human, later-developing geometrical abilities, including abilities to navigate by geometric maps (Dehaene, Izard, Pica, \& Spelke, 2006; Shusterman, Lee, \& Spelke, 2008; Spelke, Lee, \& Izard, 2010), to generate intuitions about points and lines (Izard, Pica, Spelke, \& Dehaene, in review), and to learn formal mathematics (Davis, Hersh, \& Marchisotto, 1995; Geary, 1995). Thus, future studies of the correlations and causal relations between geometrybased navigation and formal geometric reasoning may afford insight into persisting questions concerning the origins of one of our most abstract and productive systems of knowledge.
Acknowledgments We thank the members of the Laboratory for Developmental Studies at Harvard University, for help in conducting these experiments, and Ken Cheng, Anna Shusterman and George Alvarez for comments and suggestions. This research was supported by NIH grant HD-23103 to ESS and an NSF Graduate Research Fellowship to SAL.

\section{References}

Benhamou, S., \& Poucet, B. (1998). Landmark use by navigating rats (Rattus norvegicus): Contrasting geometric and featural information. Journal of Comparative Psychology, 112, 317-322.

Cartwright, B. A., \& Collett, T. S. (1982). How honey bees use landmarks to guide their return to a food source. Nature, 295, $560-564$

Cheng, K. (1986). A purely geometric module in the rats' spatial representation. Cognition, 23, 149-178.

Cheng, K. (2008). Whither geometry? Troubles of the geometric module. Trends in Cognitive Sciences, 12, 355-361.

Cheng, K., \& Gallistel, C. R. (1984). Testing the geometric power of an animal's spatial representation. In H. L. Roitblat, T. G. Bever, \& H. S. Terrace (Eds.), Animal cognition: Proceedings of the Harry Frank Guggenheim conference. Hillsdale: Erlbaum.

Cheng, K., \& Newcombe, N. S. (2005). Is there a geometric module for spatial reorientation? Squaring theory and evidence, Psychonomic Bulletin and Review, 12, 1-23.

Cressant, A., Muller, R. U., \& Poucet, B. (1997). Failure of centrally placed objects to control the firing fields of hippocampal place cells. The Journal of Neuroscience, 17, 2531-2542.

Davis, P. J., Hersh, R., \& Marchisotto, R. A. (1995). The mathematical experience. Boston: Birkhauser.

Dehaene, S., Izard, V., Pica, P., \& Spelke, E. S. (2006). Core knowledge of geometry in an Amazonian indigene group. Science, 311, 381-384.

Doeller, C. F., Barry, C., \& Burgess, N. (2010). Evidence for grid cells in a human memory network. Nature, 463, 657-661.

Doeller, C. F., \& Burgess, N. (2008). Distinct error-correcting and incidental learning of location relative to landmarks and boundaries. Proceedings of the National Academy of Sciences, 105, 5909-5914.

Foo, P., Duchon, A., Warren, W. H., \& Tarr, M. J. (2007). Humans do not switch between path knowledge and landmarks when learning a new environment. Psychological Research, 71, 240 251.

Gallistel, C. R. (1990). The organization of learning. Cambridge: MIT Press.

Geary, D. C. (1995). Reflections of evolution and culture in children's cognition: Implications for mathematical development and instruction. The American Psychologist, 50, 24-37.

Gibson, B. M., Wilks, T. J., \& Kelly, D. M. (2007). Rats (Rattus norvegicus) encode the shape of an array of discrete objects. Journal of Comparative Psychology, 121, 130-144.

Goodridge, J. P., Dudchenko, P. A., Worboys, K. A., Golob, E. J., \& Taube, J. S. (1998). Cue control and head direction cells. Behavioral Neuroscience, 112, 749-761.

Gouteux, S., \& Spelke, E. S. (2001). Children's use of geometry and landmarks to reorient in an open space. Cognition, 81, 119-148.

Hermer, L., \& Spelke, E. (1996). Modularity and development: The case of spatial reorientation. Cognition, 61, 195-232.

Huttenlocher, J., \& Lourenco, S. F. (2007). Coding location in enclosed spaces: Is geometry the principle? Developmental Science, 10, 741-746.

Izard, V., Pica, P., Spelke, E. S., \& Dehaene, S. (in review). Euclidean intuitions of geometry in an Amazonian indigene group. 
Knierim, J. J., Kudrimoti, H. S., \& McNaughton, B. L. (1995). Place cells, head direction cells, and the learning of landmark stability. The Journal of Neuroscience, 15, 1648-1659.

Learmonth, A. E., Nadel, L., \& Newcombe, N. S. (2002). Children's use of landmarks: Implications for modularity theory. Psychological Science, 13, 337-341.

Lee, S. A., Shusterman, A., \& Spelke, E. S. (2006). Reorientation and landmark-guided search by young children: Evidence for two systems. Psychological Science, 17, 577-582.

Lee, S. A., \& Spelke, E. S. (2008). Children's use of geometry for reorientation. Developmental Science, 11, 743-749.

Lee, S. A., \& Spelke, E. S. (2010). A modular geometric mechanism for navigation in disoriented children. Cognitive Psychology, 61, 152-176.

Lee, S. A., Winkler-Rhoades, N., \& Spelke, E. S. (2010). "Fooling the geometric module: Children reorient by perceived shape of the spatial layout, not by image-matching or relational information." 24th International Attention and Performance Symposium on Space, Time \& Number, Paris, France.

Lever, C., Wills, T., Cacucci, F., Burgess, N., \& O'Keefe, J. (2002). Long-term plasticity in hippocampal place-cell representation of environmental geometry. Nature, 416, 90-94.

Mackintosh, N. J. (2002). Do not ask whether they have a cognitive map, but how they find their way about. Psicológica, 23, 165-185.

O'Keefe, J., \& Burgess, N. (1996). Geometric determinants of the place fields of hippocampal neurons. Nature, 381, 425-428.

O'Keefe, J., \& Nadel, L. (1978). The Hippocampus as a cognitive map. Oxford, England: Clarendon Press.

Pearce, J. M., Graham, M., Good, M. A., Jones, P. M., \& McGregor, A. (2006). Potentiation, overshadowing, and blocking of spatial learning based on the shape of the environment. Journal of Experimental Psychology: Animal Behavior Processes, 32, 201-214.

Pecchia, T., \& Vallortigara, G. (2010). Re-orienting strategies in a rectangular array of landmarks by domestic chicks (Gallus gallus). Journal of Comparative Psychology, 124, 147-158.

Plato (ca. 380 B. C./1949). Meno (B. Jowett, trans.). Indianapolis, IN: Bobbs-Merrill.

Quirk, G. J., Muller, R. U., \& Kubie, J. L. (1990). The firing of hippocampal place cells in the dark depends on the rat's recent experience. The Journal of Neuroscience, 10, 2008-2017.
Shettleworth, S. J. (2010). Cognition, evolution, and behavior. New York: Oxford University Press.

Sheynikhovich, D., Chavarriaga, R., Strösslin, T., Arleo, A., \& Gerstner, W. (2009). Is there a geometric module for spatial orientation? Insights from a rodent navigation model. Psychological Review, 116, 540-566.

Shusterman, A., Lee, S. A., \& Spelke, E. S. (2008). Young children's spontaneous use of geometry in maps. Developmental Science, 11, F1-F7.

Solstad, T., Boccara, C. N., Kropff, E., Moser, M., \& Moser, E. I. (2008). Representation of geometric borders in the entorhinal cortex. Science, 322, 1865-1868.

Spelke, E., Lee, S. A., \& Izard, V. (2010). Beyond core knowledge: Natural geometry. Cognitive Science, 34, 863-884.

Stürzl, W., Cheung, A., Cheng, K., \& Zeil, J. (2008). The information content of panoramic Images I: The rotational errors and the similarity of views in rectangular experimental arenas. Journal of Experimental Psychology: Animal Behavior Processes, 34, 1-14.

Taube, J. S., Muller, R. U., \& Ranck, J. B. (1990). Head-direction cells recorded from the postsubiculum in freely moving rats. II. Effects of environmental manipulations. The Journal of Neuroscience, 10, 436-447.

Tolman, E. C. (1948). Cognitive maps in rats and man. Psychological Review, 55, 189-208.

Twyman, A., Friedman, A., \& Spetch, M. L. (2007). Penetrating the geometric module: Catalyzing children's use of landmarks. Developmental Psychology, 43, 1523-1530.

von Helmholtz, H. (1885/1962). Treatise on physiological optics, Vol. 3. (J. P. C. Southall, trans.). New York: Optical Society of America.

Wang, R. F., \& Spelke, E. S. (2002). Human spatial representation: Insights from animals. Trends in Cognitive Sciences, 6, 376-382.

Wehner, R., \& Menzel, R. (1990). Do insects have cognitive maps? Annual Review of Neuroscience, 13, 403-414.

Wystrach, A., \& Beugnon, G. (2009). Ants learn geometry and features. Current Biology, 19, 61-66.

Zugaro, M. B., Berthoz, A., \& Wiener, S. I. (2001). Background, but not foreground, spatial cues are taken as references for head direction responses by rat anterodorsal thalamus neurons. The Journal of Neuroscience, 21, 1-5. 